\title{
Generalized quasi-statistical structures
}

\author{
Adara M. Blaga and Antonella Nannicini
}

\begin{abstract}
Given a non-degenerate $(0,2)$-tensor field $h$ on a smooth manifold $M$, we consider a natural generalized complex and a generalized product structure on the generalized tangent bundle $T M \oplus T^{*} M$ of $M$ and we show that they are $\nabla$-integrable, for $\nabla$ an affine connection on $M$, if and only if $(M, h, \nabla)$ is a quasi-statistical manifold. We introduce the notion of generalized quasi-statistical structure and we prove that any quasi-statistical structure on $M$ induces generalized quasi-statistical structures on $T M \oplus T^{*} M$. In this context, dual connections are considered and some of their properties are established. The results are described in terms of Patterson-Walker and Sasaki metrics on $T^{*} M$, horizontal lift and Sasaki metrics on $T M$ and, when the connection $\nabla$ is flat, we define prolongation of quasi-statistical structures on manifolds to their cotangent and tangent bundles via generalized geometry. Moreover, Norden and Para-Norden structures are defined on $T^{*} M$ and $T M$.
\end{abstract}

\section{Introduction}

Statistical manifolds were introduced in [1], [7]. They are manifolds of probability distributions, used in Information Geometry and related to Codazzi tensors and Affine Geometry. Let $h$ be a pseudo-Riemannian metric and let $\nabla$ be a torsion-free affine connection on a smooth manifold $M$. Then $(M, h, \nabla)$ is called a statistical manifold if

2010 Mathematics Subject Classification. 53C15, 53B05, 53D05.

Key words and phrases. Quasi-statistical structures, generalized geometry, Patterson-Walker metric, Sasaki metric, Norden structures. 
$\left(\nabla_{X} h\right)(Y, Z)=\left(\nabla_{Y} h\right)(X, Z)$, for all $X, Y, Z \in C^{\infty}(T M)$. The definition can be extended to $(0,2)$-tensor fields and affine connections $\nabla$ with torsion, $T^{\nabla}$. In this case, $(h, \nabla)$ is called a quasi-statistical structure on $M$ if $d^{\nabla} h=0$, where $\left(d^{\nabla} h\right)(X, Y, Z):=$ $\left(\nabla_{X} h\right)(Y, Z)-\left(\nabla_{Y} h\right)(X, Z)+h\left(T^{\nabla}(X, Y), Z\right)$, for all $X, Y, Z \in C^{\infty}(T M)$, and the triple $(M, h, \nabla)$ is called a quasi-statistical manifold.

In this paper, given a non-degenerate $(0,2)$-tensor field $h$ and an affine connection $\nabla$ on a smooth manifold $M$, we consider a natural generalized complex and a generalized product structure on the generalized tangent bundle $T M \oplus T^{*} M$ of $M$ and we show that they are $\nabla$-integrable if and only if $(M, h, \nabla)$ is a quasi-statistical manifold. We introduce the notion of generalized quasi-statistical structure and we prove that any quasi-statistical structure on $M$, defined by a symmetric or skew-symmetric tensor, induces two natural generalized quasi-statistical structures on $T M \oplus T^{*} M$. We compute the dual connections and study some of their properties. The results are described in terms of Patterson-Walker and Sasaki metrics on $T^{*} M$, horizontal lift and Sasaki metrics on $T M$. In the case, the connection $\nabla$ is flat we can define prolongation of quasi-statistical structures on manifolds to their cotangent and tangent bundles via generalized geometry. Moreover, in the last section, we construct Norden and Para-Norden structures on $T^{*} M$ and $T M$.

\section{Quasi-statistical structures and generalized struc- tures induced}

Let $M$ be a smooth manifold and $h$ a non-degenerate $(0,2)$-tensor field on $M$. On the generalized tangent bundle $T M \oplus T^{*} M$ of $M$, we shall consider the generalized complex structure

$$
\hat{J}_{c}:=\left(\begin{array}{cc}
0 & -h^{-1} \\
h & 0
\end{array}\right)
$$

and the generalized product structure

$$
\hat{J}_{p}:=\left(\begin{array}{cc}
0 & h^{-1} \\
h & 0
\end{array}\right),
$$

where we denoted by $h$ the musical isomorphism, $b_{h}: T M \rightarrow T^{*} M, b_{h}(X):=i_{X} h$, and by $h^{-1}$ its inverse, $\sharp_{h}: T^{*} M \rightarrow T M$. 
Let

$$
<X+\alpha, Y+\beta>:=-\frac{1}{2}(\alpha(Y)+\beta(X))
$$

be the natural indefinite metric on $T M \oplus T^{*} M$ and

$$
(X+\alpha, Y+\beta):=-\frac{1}{2}(\alpha(Y)-\beta(X))
$$

be the natural symplectic structure on $T M \oplus T^{*} M$.

REMARK 2.1. i) If $h$ is symmetric, then:

$$
\begin{aligned}
& <\hat{J}_{c} \sigma, \hat{J}_{c} \tau>=-<\sigma, \tau>\text { and }\left(\hat{J}_{c} \sigma, \hat{J}_{c} \tau\right)=(\sigma, \tau), \\
& <\hat{J}_{p} \sigma, \hat{J}_{p} \tau>=<\sigma, \tau>\text { and }\left(\hat{J}_{p} \sigma, \hat{J}_{p} \tau\right)=-(\sigma, \tau),
\end{aligned}
$$

or, equivalently:

$$
\begin{aligned}
& <\hat{J}_{c} \sigma, \tau>=<\sigma, \hat{J}_{c} \tau>\text { and }\left(\hat{J}_{c} \sigma, \tau\right)=-\left(\sigma, \hat{J}_{c} \tau\right), \\
& <\hat{J}_{p} \sigma, \tau>=<\sigma, \hat{J}_{p} \tau>\text { and }\left(\hat{J}_{p} \sigma, \tau\right)=-\left(\sigma, \hat{J}_{p} \tau\right),
\end{aligned}
$$

for any $\sigma, \tau \in C^{\infty}\left(T M \oplus T^{*} M\right)$.

i) If $h$ is skew-symmetric, then:

$$
\begin{aligned}
& <\hat{J}_{c} \sigma, \hat{J}_{c} \tau>=<\sigma, \tau>\text { and }\left(\hat{J}_{c} \sigma, \hat{J}_{c} \tau\right)=-(\sigma, \tau), \\
& <\hat{J}_{p} \sigma, \hat{J}_{p} \tau>=-<\sigma, \tau>\text { and }\left(\hat{J}_{p} \sigma, \hat{J}_{p} \tau\right)=(\sigma, \tau),
\end{aligned}
$$

or, equivalently:

$$
\begin{aligned}
& <\hat{J}_{c} \sigma, \tau>=-<\sigma, \hat{J}_{c} \tau>\text { and }\left(\hat{J}_{c} \sigma, \tau\right)=\left(\sigma, \hat{J}_{c} \tau\right), \\
& <\hat{J}_{p} \sigma, \tau>=-<\sigma, \hat{J}_{p} \tau>\text { and }\left(\hat{J}_{p} \sigma, \tau\right)=\left(\sigma, \hat{J}_{p} \tau\right),
\end{aligned}
$$

for any $\sigma, \tau \in C^{\infty}\left(T M \oplus T^{*} M\right)$.

On $T M \oplus T^{*} M$ we consider the bilinear form:

$$
\check{h}(X+\alpha, Y+\beta):=h(X, Y)+h\left(h^{-1}(\alpha), h^{-1}(\beta)\right),
$$

for any $X, Y \in C^{\infty}(T M)$ and $\alpha, \beta \in C^{\infty}\left(T^{*} M\right)$.

A direct computation gives the following: 
LEMmA 2.2. The structures $\hat{J}_{c}$ and $\hat{J}_{p}$ satisfy respectively:

$$
\begin{gathered}
\check{h}\left(\hat{J}_{c} \sigma, \tau\right)=2(\sigma, \tau), \\
\check{h}\left(\sigma, \hat{J}_{p} \tau\right)=2<\sigma, \tau>,
\end{gathered}
$$

for any $\sigma, \tau \in C^{\infty}\left(T M \oplus T^{*} M\right)$.

For $\nabla$ an affine connection on $M$, we consider the bracket $[\cdot, \cdot]_{\nabla}$ on $C^{\infty}\left(T M \oplus T^{*} M\right)$ [6]:

$$
[X+\alpha, Y+\beta]_{\nabla}:=[X, Y]+\nabla_{X} \beta-\nabla_{Y} \alpha
$$

for all $X, Y \in C^{\infty}(T M)$ and $\alpha, \beta \in C^{\infty}\left(T^{*} M\right)$.

A generalized complex or product structure $\hat{J}$ is called $\nabla$-integrable if its Nijenhuis tensor field $N_{\hat{J}}^{\nabla}$ with respect to $\nabla$ :

$$
N_{\hat{J}}^{\nabla}(\sigma, \tau):=[\hat{J} \sigma, \hat{J} \tau]_{\nabla}-\hat{J}[\hat{J} \sigma, \tau]_{\nabla}-\hat{J}[\sigma, \hat{J} \tau]_{\nabla}+\hat{J}^{2}[\sigma, \tau]_{\nabla}
$$

vanishes for all $\sigma, \tau \in C^{\infty}\left(T M \oplus T^{*} M\right)$.

Let $M$ be a smooth manifold with a non-degenerate $(0,2)$-tensor field $h$ and an affine connection $\nabla$.

DEFINITION 2.3. [4] We call $(h, \nabla)$ a quasi-statistical structure (respectively, $(M, h, \nabla)$ a quasi-statistical manifold) if $d^{\nabla} h=0$, where

$$
\left(d^{\nabla} h\right)(X, Y, Z):=\left(\nabla_{X} h\right)(Y, Z)-\left(\nabla_{Y} h\right)(X, Z)+h\left(T^{\nabla}(X, Y), Z\right)
$$

for any $X, Y, Z \in C^{\infty}(T M)$ and $T^{\nabla}(X, Y):=\nabla_{X} Y-\nabla_{Y} X-[X, Y]$.

We can state:

Proposition 2.4. The structures $\hat{J}_{c}$ and $\hat{J}_{p}$ are integrable if and only if $(M, h, \nabla)$ is a quasi-statistical manifold.

PROOF. In this proof we will shortly denote $\hat{J}_{\mp}$ for $\hat{J}_{c}=: \hat{J}_{-}$and $\hat{J}_{p}=: \hat{J}_{+}$.

Let us compute:

$$
\begin{gathered}
N_{\hat{J}_{\mp}}^{\nabla}(X, Y)=\left[\hat{J}_{\mp} X, \hat{J}_{\mp} Y\right]_{\nabla}-\hat{J}_{\mp}\left[\hat{J}_{\mp} X, Y\right]_{\nabla}-\hat{J}_{\mp}\left[X, \hat{J}_{\mp} Y\right]_{\nabla}+\hat{J}_{\mp}^{2}[X, Y]_{\nabla}= \\
= \pm h^{-1}\left(\left(\nabla_{X} h\right) Y-\left(\nabla_{Y} h\right) X+h\left(\nabla_{X} Y-\nabla_{Y} X-[X, Y]\right)\right)=
\end{gathered}
$$




$$
\begin{aligned}
& = \pm h^{-1}\left(\left(d^{\nabla} h\right)(X, Y)\right) \\
& N_{\hat{J}_{\mp}}^{\nabla}(X, h(Y))=\left[\hat{J}_{\mp} X, \hat{J}_{\mp} h(Y)\right]_{\nabla}-\hat{J}_{\mp}\left[\hat{J}_{\mp} X, h(Y)\right]_{\nabla}-\hat{J}_{\mp}\left[X, \hat{J}_{\mp} h(Y)\right]_{\nabla}+\hat{J}_{\mp}^{2}[X, h(Y)]_{\nabla}= \\
& =\mp\left(\left(\nabla_{X} h\right) Y-\left(\nabla_{Y} h\right) X+h\left(\nabla_{X} Y-\nabla_{Y} X-[X, Y]\right)\right)= \\
& =\mp\left(d^{\nabla} h\right)(X, Y) \\
& N_{\hat{J}_{\mp}}^{\nabla}(h(X), h(Y))=\left[\hat{J}_{\mp} h(X), \hat{J}_{\mp} h(Y)\right]_{\nabla}-\hat{J}_{\mp}\left[\hat{J}_{\mp} h(X), h(Y)\right]_{\nabla}-\hat{J}_{\mp}\left[h(X), \hat{J}_{\mp} h(Y)\right]_{\nabla}+ \\
& +\hat{J}_{\mp}^{2}[h(X), h(Y)]_{\nabla}= \\
& =-h^{-1}\left(\left(\nabla_{X} h\right) Y-\left(\nabla_{Y} h\right) X+h\left(\nabla_{X} Y-\nabla_{Y} X-[X, Y]\right)\right)= \\
& =-h^{-1}\left(\left(d^{\nabla} h\right)(X, Y)\right),
\end{aligned}
$$

for any $X, Y \in C^{\infty}(T M)$. Therefore the proof is complete.

\section{$3 \quad$ Generalized quasi-statistical structures}

Definition 3.1. We call $D: C^{\infty}\left(T M \oplus T^{*} M\right) \times C^{\infty}\left(T M \oplus T^{*} M\right) \rightarrow C^{\infty}\left(T M \oplus T^{*} M\right)$ an affine connection on $T M \oplus T^{*} M$ if it is $\mathbb{R}$-bilinear and for any $f \in C^{\infty}(M)$ and $\sigma, \tau \in C^{\infty}\left(T M \oplus T^{*} M\right)$, we have:

1. $D_{f \sigma} \tau=f D_{\sigma} \tau$,

2. $D_{\sigma}(f \tau)=\sigma(f) \tau+f D_{\sigma} \tau$,

where $(X+\alpha)(f):=X(f)$, for $X+\alpha \in C^{\infty}\left(T M \oplus T^{*} M\right)$.

Let $\hat{h}$ be a non-degenerate $(0,2)$-tensor field and $D$ an affine connection on the generalized tangent bundle $T M \oplus T^{*} M$ of the smooth manifold $M$.

Definition 3.2. We call $(\hat{h}, D)$ a generalized quasi-statistical structure if $d^{D} \hat{h}=0$, where

$$
\left(d^{D} \hat{h}\right)(\sigma, \tau, \nu):=\left(D_{\sigma} \hat{h}\right)(\tau, \nu)-\left(D_{\tau} \hat{h}\right)(\sigma, \nu)+\hat{h}\left(T^{D}(\sigma, \tau), \nu\right),
$$

for any $\sigma, \tau, \nu \in C^{\infty}\left(T M \oplus T^{*} M\right)$ and $T^{D}(\sigma, \tau):=D_{\sigma} \tau-D_{\tau} \sigma-[\sigma, \tau]_{\nabla}$, with $\nabla$ a given connection on $M$. 


\subsection{Generalized quasi-statistical structures induced by quasi- statistical structures}

Let $h$ be a non-degenerate $(0,2)$-tensor field and let $\nabla$ be an affine connection on $M$. We define the affine connection $\hat{\nabla}$ on $T M \oplus T^{*} M$ by:

$$
\hat{\nabla}_{X+\alpha} Y+\beta:=\nabla_{X} Y+h\left(\nabla_{X} h^{-1}(\beta)\right),
$$

for any $X, Y \in C^{\infty}(T M)$ and $\alpha, \beta \in C^{\infty}\left(T^{*} M\right)$.

Theorem 3.3. (TM $\left.\oplus T^{*} M, \hat{h}, \hat{\nabla}\right)$ is a generalized quasi-statistical manifold if and only if $(M, h, \nabla)$ is a quasi-statistical manifold, where $\hat{h}$ is precisely $<\cdot, \cdot>$ or $(\cdot, \cdot)$ given by (3) and (4) respectively, according as $h$ is symmetric or skew-symmetric, and $\hat{\nabla}$ is given by (6).

Proof. First notice that the torsion of $\hat{\nabla}$ equals to

$$
\begin{gathered}
T^{\hat{\nabla}}(X+\alpha, Y+\beta):=\hat{\nabla}_{X+\alpha} Y+\beta-\hat{\nabla}_{Y+\beta} X+\alpha-[X+\alpha, Y+\beta]_{\nabla}= \\
=T^{\nabla}(X, Y)+h\left(\nabla_{X} h^{-1}(\beta)-\nabla_{Y} h^{-1}(\alpha)\right)-\nabla_{X} \beta+\nabla_{Y} \alpha .
\end{gathered}
$$

We have:

$$
\begin{aligned}
& \left(d^{\hat{\nabla}} \hat{h}\right)(X+\alpha, Y+\beta, Z+\gamma):=\left(\hat{\nabla}_{X+\alpha} \hat{h}\right)(Y+\beta, Z+\gamma)-\left(\hat{\nabla}_{Y+\beta} \hat{h}\right)(X+\alpha, Z+\gamma)+ \\
& +\hat{h}\left(T^{\hat{\nabla}}(X+\alpha, Y+\beta), Z+\gamma\right):= \\
& :=X(\hat{h}(Y+\beta, Z+\gamma))-\hat{h}\left(\hat{\nabla}_{X+\alpha} Y+\beta, Z+\gamma\right)-\hat{h}\left(Y+\beta, \hat{\nabla}_{X+\alpha}, Z+\gamma\right)- \\
& -Y(\hat{h}(X+\alpha, Z+\gamma))+\hat{h}\left(\hat{\nabla}_{Y+\beta} X+\alpha, Z+\gamma\right)+\hat{h}\left(X+\alpha, \hat{\nabla}_{Y+\beta}, Z+\gamma\right)+ \\
& +\hat{h}\left(T^{\hat{\nabla}}(X+\alpha, Y+\beta), Z+\gamma\right):= \\
& :=-\frac{1}{2}\left[X(\beta(Z) \pm \gamma(Y))-h\left(\nabla_{X} h^{-1}(\beta), Z\right) \mp \gamma\left(\nabla_{X} Y\right)-\beta\left(\nabla_{X} Z\right) \mp h\left(\nabla_{X} h^{-1}(\gamma), Y\right)\right]+ \\
& +\frac{1}{2}\left[Y(\alpha(Z) \pm \gamma(X))-h\left(\nabla_{Y} h^{-1}(\alpha), Z\right) \mp \gamma\left(\nabla_{Y} X\right)-\alpha\left(\nabla_{Y} Z\right) \mp h\left(\nabla_{Y} h^{-1}(\gamma), X\right)\right]- \\
& -\frac{1}{2}\left[h\left(\nabla_{X} h^{-1}(\beta), Z\right)-h\left(\nabla_{Y} h^{-1}(\alpha), Z\right)-\left(\nabla_{X} \beta\right) Z+\left(\nabla_{Y} \alpha\right) Z\right] \mp \frac{1}{2} \gamma\left(T^{\nabla}(X, Y)\right):= \\
& :=-\frac{1}{2}\left[ \pm X(\gamma(Y)) \mp \gamma\left(\nabla_{X} Y\right) \mp h\left(\nabla_{X} h^{-1}(\gamma), Y\right) \mp\right. \\
& \mp Y(\gamma(X)) \pm \gamma\left(\nabla_{Y} X\right) \pm h\left(\nabla_{Y} h^{-1}(\gamma), X\right) \pm \gamma\left(T^{\nabla}(X, Y)\right):= \\
& :=-\frac{1}{2}\left(d^{\nabla} h\right)\left(X, Y, h^{-1}(\gamma)\right) \text {. }
\end{aligned}
$$

Therefore the proof is complete. 
The couple $(\hat{h}, \hat{\nabla})$ with $\hat{h}$ given by (3) or (4) respectively (according as $h$ is symmetric or skew-symmetric) and $\hat{\nabla}$ given by (6) will be called the generalized quasi-statistical structure induced by $(h, \nabla)$.

A direct computation gives the expression of the generalized dual quasi-statistical connection of $\hat{\nabla}$, precisely:

Proposition 3.4. Let $(M, h, \nabla)$ be a quasi-statistical manifold and let $(\hat{h}, \hat{\nabla})$ be the generalized quasi-statistical structure induced on $T M \oplus T^{*} M$. Then the generalized dual quasi-statistical connection, $\hat{\nabla}^{*}$, defined by:

$$
\hat{h}\left(Y+\beta, \hat{\nabla}_{X+\alpha}^{*} Z+\gamma\right)=X(\hat{h}(Y+\beta, Z+\gamma))-\hat{h}\left(\hat{\nabla}_{X+\alpha} Y+\beta, Z+\gamma\right),
$$

for all $X, Y, Z \in C^{\infty}(T M)$ and $\alpha, \beta, \gamma \in C^{\infty}\left(T^{*} M\right)$, is given by:

$$
\hat{\nabla}_{X+\alpha}^{*} Z+\gamma=h^{-1}\left(\nabla_{X} h(Z)\right)+\nabla_{X} \gamma .
$$

Proof. From the definition of the generalized dual quasi-statistical connection and using the definition of $\hat{\nabla}$, we get:

$$
\hat{h}\left(Y+\beta, \hat{\nabla}_{X+\alpha}^{*} Z+\gamma\right)=X(\beta(Z)) \pm X(\gamma(Y))-h\left(\nabla_{X} h^{-1}(\beta), Z\right) \mp \gamma\left(\nabla_{X} Y\right),
$$

for any $X, Y, Z \in C^{\infty}(T M)$ and $\alpha, \beta, \gamma \in C^{\infty}\left(T^{*} M\right)$.

Let us denote $\hat{\nabla}_{X+\alpha}^{*} Z+\gamma=: V+\eta$. Then we have:

$$
\beta(V) \pm \eta(Y)=X(\beta(Z)) \pm X(\gamma(Y))-h\left(\nabla_{X} h^{-1}(\beta), Z\right) \mp \gamma\left(\nabla_{X} Y\right),
$$

for any $X, Y, Z \in C^{\infty}(T M)$ and $\beta, \gamma \in C^{\infty}\left(T^{*} M\right)$.

Taking $\beta:=0$, we obtain:

$$
\eta(Y)=X(\gamma(Y))-\gamma\left(\nabla_{X} Y\right):=\left(\nabla_{X} \gamma\right) Y
$$

and taking $Y:=0$, we obtain:

$$
\beta(V)=X(\beta(Z))-h\left(\nabla_{X} h^{-1}(\beta), Z\right)
$$

which is equivalent to:

$h\left(V, h^{-1}(\beta)\right)=X\left(h\left(Z, h^{-1}(\beta)\right)\right)-h\left(Z, \nabla_{X} h^{-1}(\beta)\right):=\left(\nabla_{X} h\right)\left(Z, h^{-1}(\beta)\right)+h\left(\nabla_{X} Z, h^{-1}(\beta)\right)$

and to:

$$
h(V)=\left(\nabla_{X} h\right)(Z, \cdot)+h\left(\nabla_{X} Z\right)=\nabla_{X} h(Z)
$$


and to:

$$
V=h^{-1}\left(\nabla_{X} h(Z)\right) .
$$

Therefore the proof is complete.

Proposition 3.5. Let $(M, h, \nabla)$ be a quasi-statistical manifold. Then $\hat{\nabla}^{*}$ is torsionfree.

Proof. For all $X+\alpha, Y+\beta \in C^{\infty}\left(T M \oplus T^{*} M\right)$, we have:

$$
\begin{gathered}
T^{\hat{\nabla}^{*}}(X+\alpha, Y+\beta)=\hat{\nabla}_{X+\alpha}^{*} Y+\beta-\hat{\nabla}_{Y+\beta}^{*} X+\alpha-[X+\alpha, Y+\beta]_{\nabla}= \\
=h^{-1}\left(\nabla_{X} h(Y)\right)-h^{-1}\left(\nabla_{Y} h(X)\right)-[X, Y]= \\
=h^{-1}\left(\left(\nabla_{X} h\right) Y-\left(\nabla_{Y} h\right) X+h\left(T^{\nabla}(X, Y)\right)\right)= \\
=h^{-1}\left(d^{\nabla}(X, Y)\right)=0 .
\end{gathered}
$$

Let $h$ be a non-degenerate, symmetric or skew-symmetric $(0,2)$-tensor field on $M$ and let $\nabla$ be an affine connection on $M$. We have the following:

THEOREM 3.6. (TM $\left.\oplus T^{*} M, \breve{h}, \hat{\nabla}\right)$ is a generalized quasi-statistical manifold if and only if $(M, h, \nabla)$ is a quasi-statistical manifold, where $\breve{h}$ is given by (5) and $\hat{\nabla}$ is given by (6).

Proof. We have:

$$
\begin{aligned}
&\left(d^{\hat{\nabla}} \check{h}\right)(X+\alpha, Y+\beta, Z+\gamma):=\left(\hat{\nabla}_{X+\alpha} \check{h}\right)(Y+\beta, Z+\gamma)-\left(\hat{\nabla}_{Y+\beta} \check{h}\right)(X+\alpha, Z+\gamma)++\check{h}\left(T^{\hat{\nabla}}(X+\alpha, Y+\beta), Z+\gamma\right):= \\
&:=X(\check{h}(Y+\beta, Z+\gamma))-\check{h}\left(\hat{\nabla}_{X+\alpha} Y+\beta, Z+\gamma\right)-\check{h}\left(Y+\beta, \hat{\nabla}_{X+\alpha}, Z+\gamma\right)- \\
&-Y(\check{h}(X+\alpha, Z+\gamma))+\check{h}\left(\hat{\nabla}_{Y+\beta} X+\alpha, Z+\gamma\right)+\check{h}\left(X+\alpha, \hat{\nabla}_{Y+\beta}, Z+\gamma\right)+ \\
&+\check{h}\left(T^{\hat{\nabla}}(X+\alpha, Y+\beta), Z+\gamma\right):= \\
&:=X(h(Y, Z))+X\left(\beta\left(h^{-1}(\gamma)\right)\right)-\check{h}\left(\nabla_{X} Y+h\left(\nabla_{X} h^{-1}(\beta)\right), Z+\gamma\right)- \\
&-\check{h}\left(Y+\beta, \nabla_{X} Z+h\left(\nabla_{X} h^{-1}(\gamma)\right)\right)- \\
&-Y(h(X, Z))-Y\left(\alpha\left(h^{-1}(\gamma)\right)\right)+\check{h}\left(\nabla_{Y} X+h\left(\nabla_{Y} h^{-1}(\alpha)\right), Z+\gamma\right)+
\end{aligned}
$$




$$
\begin{gathered}
+\breve{h}\left(X+\alpha, \nabla_{Y} Z+h\left(\nabla_{Y} h^{-1}(\gamma)\right)\right)+h\left(T^{\nabla}(X, Y), Z\right) \pm \gamma\left(\left(\nabla_{X} h^{-1}\right) \beta-\left(\nabla_{Y} h^{-1}\right) \alpha\right):= \\
:=\left(d^{\nabla} h\right)(X, Y, Z)+\left(\nabla_{X} \beta\right) h^{-1}(\gamma)-\left(\nabla_{Y} \alpha\right) h^{-1}(\gamma) \pm \gamma\left(h^{-1}\left(\nabla_{Y} \alpha\right)-h^{-1}\left(\nabla_{X} \beta\right)\right):= \\
:=\left(d^{\nabla} h\right)(X, Y, Z)
\end{gathered}
$$

where the sign + is for $h$ symmetric, - is for $h$ skew-symmetric. Therefore the proof is complete.

Proposition 3.7. Let $(M, h, \nabla)$ be a quasi-statistical manifold and let $(\check{h}, \hat{\nabla})$ be the generalized quasi-statistical structure induced on $T M \oplus T^{*} M$. Then the generalized dual quasi-statistical connection, $\hat{\nabla}_{\breve{h}}^{*}$, defined by:

$$
\check{h}\left(Y+\beta,\left(\hat{\nabla}_{\breve{h}}^{*}\right)_{X+\alpha} Z+\gamma\right)=X(\check{h}(Y+\beta, Z+\gamma))-\check{h}\left(\hat{\nabla}_{X+\alpha} Y+\beta, Z+\gamma\right),
$$

for all $X, Y, Z \in C^{\infty}(T M)$ and $\alpha, \beta, \gamma \in C^{\infty}\left(T^{*} M\right)$, is given by:

$$
\left(\hat{\nabla}_{\breve{h}}^{*}\right)_{X+\alpha} Z+\gamma=h^{-1}\left(\nabla_{X} h(Z)\right)+\nabla_{X} \gamma
$$

Therefore:

$$
\hat{\nabla}_{\breve{h}}^{*}=\hat{\nabla}^{*}
$$

Proof. We get:

$$
\begin{gathered}
\check{h}\left(Y+\beta,\left(\hat{\nabla}_{\breve{h}}^{*}\right)_{X+\alpha} Z+\gamma\right)=X(h(Y, Z))+X\left(\beta\left(h^{-1}(\gamma)\right)\right)-h\left(\nabla_{X} Y, Z\right) \mp \gamma\left(\nabla_{X} h^{-1}(\beta)\right)= \\
=X(h(Y, Z))-h\left(\nabla_{X} Y, Z\right) \mp \gamma\left(\nabla_{X} h^{-1}(\beta)\right) \pm X\left(\gamma\left(h^{-1}(\beta)\right)\right),
\end{gathered}
$$

for any $X, Y, Z \in C^{\infty}(T M)$ and $\alpha, \beta, \gamma \in C^{\infty}\left(T^{*} M\right)$.

Let us denote $\left(\hat{\nabla}_{\breve{h}}^{*}\right)_{X+\alpha} Z+\gamma=: V+\eta$. Then we have:

$$
h(Y, V) \pm \eta\left(h^{-1}(\beta)\right)=X(h(Y, Z))-h\left(\nabla_{X} Y, Z\right) \pm\left(\nabla_{X} \gamma\right) h^{-1}(\beta)
$$

for any $X, Y, Z \in C^{\infty}(T M)$ and $\beta \in C^{\infty}\left(T^{*} M\right)$.

Taking $Y:=0$, we obtain:

$$
\eta\left(h^{-1}(\beta)\right)=\nabla_{X} h^{-1}(\beta)
$$

and taking $\beta:=0$, we obtain:

$$
h(Y, V)=\left(\nabla_{X} h\right)(Y, Z)+h\left(Y, \nabla_{X} Z\right)
$$


which is equivalent to:

$$
h(V)=\left(\nabla_{X} h\right)(Z, \cdot)+h\left(\nabla_{X} Z\right)=\nabla_{X} h(Z)
$$

and to:

$$
V=h^{-1}\left(\nabla_{X} h(Z)\right)
$$

Therefore the proof is complete.

Given an affine connection $D$ on $T M \oplus T^{*} M$, we define the curvature operator of $D$, $R^{D}: C^{\infty}\left(T M \oplus T^{*} M\right) \times C^{\infty}\left(T M \oplus T^{*} M\right) \times C^{\infty}\left(T M \oplus T^{*} M\right) \rightarrow C^{\infty}\left(T M \oplus T^{*} M\right)$, on $\sigma, \tau, \nu \in C^{\infty}\left(T M \oplus T^{*} M\right)$, as in the following:

$$
R^{D}(\sigma, \tau) \nu=\left(D_{\sigma} D_{\tau}-D_{\tau} D_{\sigma}-D_{[\sigma, \tau]_{\nabla}}\right) \nu
$$

where $\nabla$ is a given connection on $M$.

Proposition 3.8. Let $(M, h, \nabla)$ be a quasi-statistical manifold and let $(\hat{h}, \hat{\nabla})$ be the generalized quasi-statistical structure induced on $T M \oplus T^{*} M$. Then the curvature operators of $\hat{\nabla}$ and $\hat{\nabla}^{*}$ are given respectively by:

$$
\begin{gathered}
R^{\hat{\nabla}}(X+\alpha, Y+\beta) Z+\gamma=R^{\nabla}(X, Y) Z+h\left(R^{\nabla}(X, Y) h^{-1}(\gamma)\right) \\
R^{\hat{\nabla}^{*}}(X+\alpha, Y+\beta) Z+\gamma=h^{-1}\left(R^{\nabla}(X, Y) h(Z)\right)+R^{\nabla}(X, Y) \gamma
\end{gathered}
$$

where $X, Y, Z \in C^{\infty}(T M), \alpha, \beta, \gamma \in C^{\infty}\left(T^{*} M\right)$ and $R^{\nabla}$ is the curvature operator of $\nabla$. In particular, $\hat{\nabla}$ and its dual $\hat{\nabla}^{*}$ are flat if and only if $\nabla$ is flat.

ProOF. Let us compute:

$$
\begin{aligned}
\hat{\nabla}_{X+\alpha} \hat{\nabla}_{Y+\beta} Z+\gamma-\hat{\nabla}_{Y+\beta} \hat{\nabla}_{X+\alpha} Z+\gamma-\hat{\nabla}_{[X+\alpha, Y+\beta]_{\nabla}} Z+\gamma:= \\
:=\hat{\nabla}_{X+\alpha}\left(\nabla_{Y} Z+h\left(\nabla_{Y} h^{-1}(\gamma)\right)\right)-\hat{\nabla}_{Y+\beta}\left(\nabla_{X} Z+h\left(\nabla_{X} h^{-1}(\gamma)\right)\right)- \\
-\nabla_{[X, Y]} Z-h\left(\nabla_{[X, Y]} h^{-1}(\gamma)\right):= \\
:=\nabla_{X} \nabla_{Y} Z+h\left(\nabla_{X} \nabla_{Y} h^{-1}(\gamma)\right)-\nabla_{Y} \nabla_{X} Z-h\left(\nabla_{Y} \nabla_{X} h^{-1}(\gamma)\right)- \\
-\nabla_{[X, Y]} Z-h\left(\nabla_{[X, Y]} h^{-1}(\gamma)\right):= \\
:=R^{\nabla}(X, Y) Z+h\left(R^{\nabla}(X, Y) h^{-1}(\gamma)\right)
\end{aligned}
$$

and:

$$
\hat{\nabla}_{X+\alpha}^{*} \hat{\nabla}_{Y+\beta}^{*} Z+\gamma-\hat{\nabla}_{Y+\beta}^{*} \hat{\nabla}_{X+\alpha}^{*} Z+\gamma-\hat{\nabla}_{[X+\alpha, Y+\beta]_{\nabla}}^{*} Z+\gamma:=
$$




$$
\begin{gathered}
:=\hat{\nabla}_{X+\alpha}^{*}\left(h^{-1}\left(\nabla_{Y} h(Z)\right)+\nabla_{Y} \gamma\right)-\hat{\nabla}_{Y+\beta}^{*}\left(h^{-1}\left(\nabla_{X} h(Z)\right)+\nabla_{X} \gamma\right)- \\
-h^{-1}\left(\nabla_{[X, Y]} h(Z)\right)-\nabla_{[X, Y]} \gamma:= \\
:=h^{-1}\left(\nabla_{X} \nabla_{Y} h(Z)\right)+\nabla_{X} \nabla_{Y} \gamma-h^{-1}\left(\nabla_{Y} \nabla_{X} h(Z)\right)-\nabla_{Y} \nabla_{X} \gamma- \\
-h^{-1}\left(\nabla_{[X, Y]} h(Z)\right)-\nabla_{[X, Y]} \gamma:= \\
:=h^{-1}\left(R^{\nabla}(X, Y) h(Z)\right)+R^{\nabla}(X, Y) \gamma .
\end{gathered}
$$

Therefore the proof is complete.

Proposition 3.9. The structures $\hat{J}_{c}$ and $\hat{J}_{p}$ are $\hat{\nabla}$-parallel and $\hat{\nabla}^{*}$-parallel.

PROOF. In this proof we will shortly denote $\hat{J}_{\mp}$ for $\hat{J}_{c}=: \hat{J}_{-}$and $\hat{J}_{p}=: \hat{J}_{+}$. Let us compute:

$$
\begin{aligned}
& \left(\hat{\nabla}_{X+\alpha} \hat{J}_{\mp}\right) Y+\beta:=\hat{\nabla}_{X+\alpha}\left(\mp h^{-1}(\beta)+h(Y)\right)-\hat{J}_{\mp}\left(\hat{\nabla}_{X+\alpha} Y+\beta\right):= \\
& :=\mp \nabla_{X} h^{-1}(\beta)+h\left(\nabla_{X} h^{-1}(h(Y))\right)-\hat{J}_{\mp}\left(\nabla_{X} Y+h\left(\nabla_{X} h^{-1}(\beta)\right)\right):= \\
& :=\mp \nabla_{X} h^{-1}(\beta)+h\left(\nabla_{X} Y\right) \pm h^{-1}\left(h\left(\nabla_{X} h^{-1}(\beta)\right)\right)-h\left(\nabla_{X} Y\right)=0 ;
\end{aligned}
$$

moreover:

$$
\begin{gathered}
\left(\hat{\nabla}_{X+\alpha}^{*} \hat{J}_{\mp}\right) Y+\beta:=\hat{\nabla}_{X+\alpha}^{*}\left(\mp h^{-1}(\beta)+h(Y)\right)-\hat{J}_{\mp}\left(\hat{\nabla}_{X+\alpha}^{*} Y+\beta\right):= \\
:=\mp h^{-1}\left(\nabla_{X} \beta\right)+\nabla_{X} h(Y)-\hat{J}_{\mp}\left(h^{-1}\left(\nabla_{X} h(Y)\right)+\nabla_{X} \beta\right):= \\
:=\mp h^{-1}\left(\nabla_{X} \beta\right)+\nabla_{X} h(Y)-\nabla_{X} h(Y) \pm h^{-1}\left(\nabla_{X} \beta\right)=0,
\end{gathered}
$$

for any $X, Y \in C^{\infty}(T M)$ and $\alpha, \beta \in C^{\infty}\left(T^{*} M\right)$. Therefore the proof is complete.

\subsection{Generalized quasi-statistical structures induced by torsion- free connections}

Another affine connection on the generalized tangent bundle $T M \oplus T^{*} M$ is naturally defined by an affine connection $\nabla$ on $M$ by:

$$
\check{\nabla}_{X+\alpha} Y+\beta:=\nabla_{X} Y+\nabla_{X} \beta
$$

for any $X, Y \in C^{\infty}(T M)$ and $\alpha, \beta \in C^{\infty}\left(T^{*} M\right)$. 
REMARK 3.10. One can check that if $h$ is a non-degenerate $(0,2)$-tensor field on $M$ which is $\nabla$-parallel, then the connections $\hat{\nabla}$ and $\check{\nabla}$ coincide (since we have $\hat{\nabla}_{X+\alpha} Y+\beta-$ $\check{\nabla}_{X+\alpha} Y+\beta=-\left(\nabla_{X} h\right)\left(h^{-1}(\beta), \cdot\right)$, for any $X, Y \in C^{\infty}(T M)$ and $\left.\alpha, \beta \in C^{\infty}\left(T^{*} M\right)\right)$. In particular, $\hat{\nabla}^{*}=\hat{\nabla}=\check{\nabla}$.

We have the following:

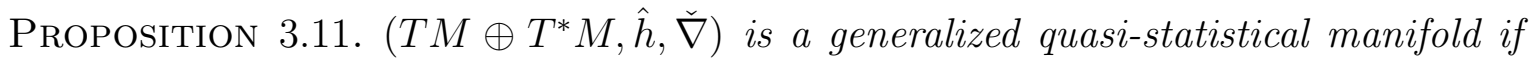
and only if $\nabla$ is torsion-free, where $\hat{h}$ is precisely $\langle\cdot, \cdot\rangle$ or $(\cdot, \cdot)$ given by (3) and (4) respectively and $\check{\nabla}$ is given by (7).

ProOF. First notice that the torsion of $\check{\nabla}$ equals to

$$
T^{\check{\nabla}}(X+\alpha, Y+\beta):=\check{\nabla}_{X+\alpha} Y+\beta-\check{\nabla}_{Y+\beta} X+\alpha-[X+\alpha, Y+\beta]_{\nabla}=T^{\nabla}(X, Y) .
$$

We have:

$$
\begin{aligned}
& \left(d^{\check{\nabla}} \hat{h}\right)(X+\alpha, Y+\beta, Z+\gamma):=\left(\check{\nabla}_{X+\alpha} \hat{h}\right)(Y+\beta, Z+\gamma)-\left(\check{\nabla}_{Y+\beta} \hat{h}\right)(X+\alpha, Z+\gamma)+ \\
& +\hat{h}\left(T^{\check{\nabla}}(X+\alpha, Y+\beta), Z+\gamma\right):= \\
& :=X(\hat{h}(Y+\beta, Z+\gamma))-\hat{h}\left(\check{\nabla}_{X+\alpha} Y+\beta, Z+\gamma\right)-\hat{h}\left(Y+\beta, \check{\nabla}_{X+\alpha}, Z+\gamma\right)- \\
& -Y(\hat{h}(X+\alpha, Z+\gamma))+\hat{h}\left(\check{\nabla}_{Y+\beta} X+\alpha, Z+\gamma\right)+\hat{h}\left(X+\alpha, \check{\nabla}_{Y+\beta}, Z+\gamma\right)+ \\
& +\hat{h}\left(T^{\check{\nabla}}(X+\alpha, Y+\beta), Z+\gamma\right):= \\
& :=-\frac{1}{2}\left[X(\beta(Z) \pm \gamma(Y))-\left(\nabla_{X} \beta\right) Z \mp \gamma\left(\nabla_{X} Y\right)-\beta\left(\nabla_{X} Z\right) \mp\left(\nabla_{X} \gamma\right) Y\right]+ \\
& +\frac{1}{2}\left[Y(\alpha(Z) \pm \gamma(X))-\left(\nabla_{Y} \alpha\right) Z \mp \gamma\left(\nabla_{Y} X\right)-\alpha\left(\nabla_{Y} Z\right) \mp\left(\nabla_{Y} \gamma\right) X\right] \mp \frac{1}{2} \gamma\left(T^{\nabla}(X, Y)\right):= \\
& :=-\frac{1}{2}\left[X(\beta(Z)) \pm X(\gamma(Y))-X(\beta(Z))+\beta\left(\nabla_{X} Z\right) \mp \gamma\left(\nabla_{X} Y\right)-\beta\left(\nabla_{X} Z\right) \mp X(\gamma(Y)) \pm \gamma\left(\nabla_{X} Y\right)\right]+ \\
& +\frac{1}{2}\left[Y(\alpha(Z)) \pm Y(\gamma(X))-Y(\alpha(Z))+\alpha\left(\nabla_{Y} Z\right) \mp \gamma\left(\nabla_{Y} X\right)-\alpha\left(\nabla_{Y} Z\right) \mp Y(\gamma(X)) \pm \gamma\left(\nabla_{Y} X\right)\right] \mp \\
& \mp \frac{1}{2} \gamma\left(T^{\nabla}(X, Y)\right)=\mp \frac{1}{2} \gamma\left(T^{\nabla}(X, Y)\right) .
\end{aligned}
$$

Therefore the proof is complete.

Proposition 3.12. Let $\nabla$ be a torsion-free affine connection on $M$ and let $(<\cdot, \cdot>, \check{\nabla})$ and $((\cdot, \cdot), \check{\nabla})$ be the canonical generalized quasi-statistical structures defined in Proposition 3.11. Then $\check{\nabla}$ and its generalized dual quasi-statistical connection, $\check{\nabla}^{*}$, coincide. 
Proof. Let us denote $\check{\nabla}_{X+\alpha}^{*} Z+\gamma=: V+\eta$. From the definition of the generalized dual quasi-statistical connection and using the definition of $\check{\nabla}$, we get:

$$
\beta(V) \pm \eta(Y)=X(\beta(Z)) \pm X(\gamma(Y))-\left(\nabla_{X} \beta\right) Z \mp \gamma\left(\nabla_{X} Y\right)
$$

for any $X, Y, Z \in C^{\infty}(T M)$ and $\beta, \gamma \in C^{\infty}\left(T^{*} M\right)$.

Taking $\beta:=0$, we obtain:

$$
\pm \eta(Y)= \pm X(\gamma(Y)) \mp \gamma\left(\nabla_{X} Y\right):= \pm\left(\nabla_{X} \gamma\right) Y
$$

and taking $Y:=0$, we obtain:

$$
\beta(V)=X(\beta(Z))-\left(\nabla_{X} \beta\right) Z:=\beta\left(\nabla_{X} Z\right)
$$

Therefore the proof is complete.

Proposition 3.13. If $\nabla$ is a torsion-free affine connection and $h$ is a $\nabla$-parallel $(0,2)$-tensor field on $M$, then $(\check{h}, \check{\nabla})$ is a generalized quasi-statistical structure, where $\check{h}$ is given by (5) and $\check{\nabla}$ is given by (7).

Proof. We have:

$$
\begin{gathered}
\left(d^{\check{\nabla} \check{h}}\right)(X+\alpha, Y+\beta, Z+\gamma):=\left(\check{\nabla}_{X+\alpha} \check{h}\right)(Y+\beta, Z+\gamma)-\left(\check{\nabla}_{Y+\beta} \check{h}\right)(X+\alpha, Z+\gamma)+ \\
+\check{h}\left(T^{\check{\nabla}}(X+\alpha, Y+\beta), Z+\gamma\right):= \\
:=X(\check{h}(Y+\beta, Z+\gamma))-\check{h}\left(\check{\nabla}_{X+\alpha} Y+\beta, Z+\gamma\right)-\check{h}\left(Y+\beta, \check{\nabla}_{X+\alpha}, Z+\gamma\right)- \\
-Y(\check{h}(X+\alpha, Z+\gamma))+\check{h}\left(\check{\nabla}_{Y+\beta} X+\alpha, Z+\gamma\right)+\check{h}\left(X+\alpha, \check{\nabla}_{Y+\beta}, Z+\gamma\right)+ \\
+\check{h}\left(T^{\check{\nabla}}(X+\alpha, Y+\beta), Z+\gamma\right):= \\
:=X\left(h(Y, Z)+h\left(h^{-1}(\beta), h^{-1}(\gamma)\right)\right)- \\
-h\left(\nabla_{X} Y, Z\right)-h\left(h^{-1}\left(\nabla_{X} \beta\right), h^{-1}(\gamma)\right)-h\left(Y, \nabla_{X} Z\right)-h\left(h^{-1}(\beta), h^{-1}\left(\nabla_{X} \gamma\right)\right)- \\
-Y\left(h(X, Z)+h\left(h^{-1}(\alpha), h^{-1}(\gamma)\right)\right)+ \\
+h\left(\nabla_{Y} X, Z\right)+h\left(h^{-1}\left(\nabla_{Y} \alpha\right), h^{-1}(\gamma)\right)+h\left(X, \nabla_{Y} Z\right)+h\left(h^{-1}(\alpha), h^{-1}\left(\nabla_{Y} \gamma\right)\right)+h\left(T^{\nabla}(X, Y), Z\right):= \\
:=\left(\nabla_{X} h\right)(Y, Z)-\left(\nabla_{Y} h\right)(X, Z)+h\left(T^{\nabla}(X, Y), Z\right)+ \\
+X\left(\beta\left(h^{-1}(\gamma)\right)\right)-\left(\nabla_{X} \beta\right) h^{-1}(\gamma)-\beta\left(h^{-1}\left(\nabla_{X} \gamma\right)\right)- \\
-Y\left(\alpha\left(h^{-1}(\gamma)\right)\right)+\left(\nabla_{Y} \alpha\right) h^{-1}(\gamma)+\alpha\left(h^{-1}\left(\nabla_{Y} \gamma\right)\right)=
\end{gathered}
$$




$$
\begin{gathered}
=\left(\nabla_{X} h\right)(Y, Z)-\left(\nabla_{Y} h\right)(X, Z)+h\left(T^{\nabla}(X, Y), Z\right)+ \\
+\beta\left(\nabla_{X} h^{-1}(\gamma)\right)-\beta\left(h^{-1}\left(\nabla_{X} \gamma\right)\right)-\alpha\left(\nabla_{Y} h^{-1}(\gamma)\right)+\alpha\left(h^{-1}\left(\nabla_{Y} \gamma\right)\right) .
\end{gathered}
$$

Also, for any $V \in C^{\infty}(T M)$, we have:

$$
\begin{gathered}
h\left(h^{-1}\left(\nabla_{X} \gamma\right)-\nabla_{X} h^{-1}(\gamma), V\right)=h\left(h^{-1}\left(\nabla_{X} \gamma\right), V\right)-h\left(\nabla_{X} h^{-1}(\gamma), V\right)= \\
=\left(\nabla_{X} \gamma\right) V-h\left(\nabla_{X} h^{-1}(\gamma), V\right):=X(\gamma(V))-\gamma\left(\nabla_{X} V\right)-h\left(\nabla_{X} h^{-1}(\gamma), V\right)= \\
=X\left(h\left(h^{-1}(\gamma), V\right)\right)-h\left(h^{-1}(\gamma), \nabla_{X} V\right)-h\left(\nabla_{X} h^{-1}(\gamma), V\right):=\left(\nabla_{X} h\right)\left(h^{-1}(\gamma), V\right)=0,
\end{gathered}
$$

hence $h^{-1}\left(\nabla_{X} \gamma\right)-\nabla_{X} h^{-1}(\gamma)=0$, for any $X \in C^{\infty}(T M)$ and $\gamma \in C^{\infty}\left(T^{*} M\right)$. Therefore, $d^{\check{\nabla} \breve{h}}=0$ and the proof is complete.

Proposition 3.14. Let $(M, h, \nabla)$ be a quasi-statistical manifold with $\nabla$ a torsion-free affine connection, $h$ a $\nabla$-parallel $(0,2)$-tensor field on $M$ and let $(\check{h}, \check{\nabla})$ be the generalized quasi-statistical structure on $T M \oplus T^{*} M$, with $\breve{h}$ given by (5) and $\check{\nabla}$ given by (7). Then $\check{\nabla}$ and its generalized dual quasi-statistical connection, $\check{\nabla}_{\breve{h}}^{*}$, coincide.

Proof. We get:

$$
\begin{gathered}
\check{h}\left(Y+\beta,\left(\check{\nabla}_{\breve{h}}^{*}\right)_{X+\alpha} Z+\gamma\right)=X(h(Y, Z))+X\left(h\left(h^{-1}(\beta), h^{-1}(\gamma)\right)\right)- \\
-h\left(\nabla_{X} Y, Z\right)-h\left(h^{-1}\left(\nabla_{X} \beta\right), h^{-1}(\gamma)\right)= \\
=h\left(Y, \nabla_{X} Z\right)+\beta\left(\nabla_{X} h^{-1}(\gamma)\right),
\end{gathered}
$$

for any $X, Y, Z \in C^{\infty}(T M)$ and $\alpha, \beta, \gamma \in C^{\infty}\left(T^{*} M\right)$.

Let us denote $\left(\check{\nabla}_{\breve{h}}^{*}\right)_{X+\alpha} Z+\gamma=: V+\eta$. Then we have:

$$
h(Y, V)+h\left(h^{-1}(\beta), h^{-1}(\eta)\right)=h\left(Y, \nabla_{X} Z\right)+\beta\left(\nabla_{X} h^{-1}(\gamma)\right),
$$

for any $X, Y, Z \in C^{\infty}(T M)$ and $\beta, \gamma \in C^{\infty}\left(T^{*} M\right)$.

Taking $Y:=0$, we obtain:

$$
\beta\left(h^{-1}(\eta)\right)=\beta\left(\nabla_{X} h^{-1}(\gamma)\right)
$$

which is equivalent to:

$$
\eta=h\left(\nabla_{X} h^{-1}(\gamma)\right)
$$

and taking $\beta:=0$, we obtain:

$$
h(Y, V)=h\left(Y, \nabla_{X} Z\right)
$$

which is equivalent to:

$$
V=\nabla_{X} Z
$$

From Remark 3.10 we get $\check{\nabla}_{\breve{h}}^{*}=\hat{\nabla}=\check{\nabla}$. Therefore the proof is complete. 


\section{The pull-back tensors on $T M \oplus T^{*} M$ of horizontal lifts, Sasaki and Patterson-Walker metrics}

\subsection{Patterson-Walker and Sasaki metrics on $T^{*} M$}

Let $M$ be a smooth manifold and let $\nabla$ be an affine connection on $M$.

Let $\pi: T^{*} M \rightarrow M$ be the canonical projection and $\pi_{*}: T\left(T^{*} M\right) \rightarrow T M$ be the tangent map of $\pi$. If $a \in T^{*} M$ and $A \in T_{a}\left(T^{*} M\right)$, then $\pi_{*}(A) \in T_{\pi(a)} M$ and we denote by $\chi_{a}$ the standard identification between $T_{\pi(a)}^{*} M$ and its tangent space $T_{a}\left(T_{\pi(a)}^{*} M\right)$.

Let $\Phi^{\nabla}: T M \oplus T^{*} M \rightarrow T\left(T^{*} M\right)$ be the bundle morphism defined by [5]:

$$
\Phi^{\nabla}(X+\alpha):=X_{a}^{H}+\chi_{a}(\alpha)
$$

where $a \in T^{*} M$ and $X_{a}^{H}$ is the horizontal lift of $X \in T_{\pi(a)} M$.

Let $\left\{x^{1}, \ldots, x^{n}\right\}$ be local coordinates on $M$, let $\left\{\tilde{x}^{1}, \ldots, \tilde{x}^{n}, y_{1}, \ldots, y_{n}\right\}$ be respectively the corresponding local coordinates on $T^{*} M$ and let $\left\{X_{1}, \ldots, X_{n}, \frac{\partial}{\partial y_{1}}, . ., \frac{\partial}{\partial y_{n}}\right\}$ be a local frame on $T\left(T^{*} M\right)$, where $X_{i}=\frac{\partial}{\partial \tilde{x}^{i}}$. The horizontal lift of $\frac{\partial}{\partial x^{i}}$ is defined by:

$$
\left(\frac{\partial}{\partial x^{i}}\right)^{H}:=X_{i}+y_{k} \Gamma_{i l}^{k} \frac{\partial}{\partial y_{l}}
$$

and we will denote $X_{i}^{H}=:\left(\frac{\partial}{\partial x^{i}}\right)^{H}$. Moreover, the vertical lift of $\frac{\partial}{\partial x^{i}}$ is defined by:

$$
\left(\frac{\partial}{\partial x^{i}}\right)^{V}:=\frac{\partial}{\partial y_{i}}
$$

where $i, k, l$ run from 1 to $n$ and $\Gamma_{i l}^{k}$ are the Christoffel's symbols of $\nabla$.

Let $\Phi^{\nabla}: T M \oplus T^{*} M \rightarrow T\left(T^{*} M\right)$ be the bundle morphism defined before (which is an isomorphism on the fibres). In local coordinates, we have the following expressions:

$$
\begin{gathered}
\Phi^{\nabla}\left(\frac{\partial}{\partial x^{i}}\right)=X_{i}^{H} \\
\Phi^{\nabla}\left(d x^{j}\right)=\frac{\partial}{\partial y_{j}} .
\end{gathered}
$$

In [9], starting from a torsion-free affine connection on $M$, the Patterson-Walker metric, $\tilde{h}$, on $T^{*} M$ is defined as in the following:

$$
\tilde{h}\left(X^{H}, Y^{H}\right)=0
$$




$$
\begin{gathered}
\tilde{h}\left(X^{V}, Y^{V}\right)=0 \\
\tilde{h}\left(Y^{V}, X^{H}\right)=\tilde{h}\left(X^{H}, Y^{V}\right)=\left(\left(\Phi^{\nabla}\right)^{-1}\left(Y^{V}\right)\right)(X),
\end{gathered}
$$

where $X, Y \in C^{\infty}\left(T^{*} M\right), X^{H}, Y^{H}$ are the horizontal lifts and $X^{V}, Y^{V}$ are the vertical lifts of $X, Y$ respectively.

The definition can also be given if $\nabla$ has torsion and we define $\tilde{h}_{ \pm}$on $T^{*} M$ as in the following:

$$
\begin{gathered}
\tilde{h}_{ \pm}\left(X^{H}, Y^{H}\right)=0 \\
\tilde{h}_{ \pm}\left(X^{V}, Y^{V}\right)=0 \\
\tilde{h}_{ \pm}\left(Y^{V}, X^{H}\right)=\left(\left(\Phi^{\nabla}\right)^{-1}\left(Y^{V}\right)\right)(X) \\
\tilde{h}_{ \pm}\left(X^{H}, Y^{V}\right)= \pm\left(\left(\Phi^{\nabla}\right)^{-1}\left(Y^{V}\right)\right)(X),
\end{gathered}
$$

where $X, Y \in C^{\infty}\left(T^{*} M\right), X^{H}, Y^{H}$ are the horizontal lifts and $X^{V}, Y^{V}$ are the vertical lifts of $X, Y$ respectively.

We denote by $\tilde{\tilde{h}}_{ \pm}$the pull-back tensors of $\tilde{h}_{ \pm}$on $T M \oplus T^{*} M$ :

$$
\tilde{\tilde{h}}_{ \pm}(\sigma, \tau):=\left(\Phi^{\nabla}\right)^{*}\left(\tilde{h}_{ \pm}\right)(\sigma, \tau):=\tilde{h}_{ \pm}\left(\Phi^{\nabla}(\sigma), \Phi^{\nabla}(\tau)\right)
$$

for any $\sigma, \tau \in C^{\infty}\left(T M \oplus T^{*} M\right)$. Remark that $\tilde{\tilde{h}}_{ \pm}$are related to the indefinite metric or to the symplectic structure of $T M \oplus T^{*} M$ as follows.

Proposition 4.1.

$$
\begin{gathered}
\tilde{\tilde{h}}_{+}=-2<\cdot, \cdot> \\
\tilde{\tilde{h}}_{-}=-2(\cdot, \cdot) .
\end{gathered}
$$

Proof. Let $\sigma=X+\alpha, \tau=Y+\beta, X, Y \in C^{\infty}(T M), \alpha, \beta \in C^{\infty}\left(T^{*} M\right)$. Then:

$$
\begin{gathered}
\tilde{\tilde{h}}_{ \pm}(\sigma, \tau)=\tilde{h}_{ \pm}\left(X^{H}+\Phi^{\nabla}(\alpha), Y^{H}+\Phi^{\nabla}(\beta)\right)= \\
=\tilde{h}_{ \pm}\left(\Phi^{\nabla}(\alpha), Y^{H}\right)+\tilde{h}_{ \pm}\left(X^{H}, \Phi^{\nabla}(\beta)\right)= \\
=\alpha(Y) \pm \beta(X) .
\end{gathered}
$$

Then we get the statement. 
Let $h$ be a non-degenerate $(0,2)$-tensor field on $M$. The Sasaki $(0,2)$-tensor field $h^{S^{*}}$ on $T^{*} M$, with respect to $\nabla$, is naturally defined by:

$$
\begin{gathered}
h^{S^{*}}\left(X^{H}, Y^{H}\right)=h(X, Y) \\
h^{S^{*}}\left(\alpha^{V}, \beta^{V}\right)=h\left(h^{-1}(\alpha), h^{-1}(\beta)\right) \\
h^{S^{*}}\left(\alpha^{V}, Y^{H}\right)=0,
\end{gathered}
$$

where $X, Y \in C^{\infty}(T M), \alpha, \beta \in C^{\infty}\left(T^{*} M\right), X^{H}, Y^{H}$ are the horizontal lifts of $X, Y$ and $\alpha^{V}, \beta^{V}$ are the vertical lifts of $\alpha, \beta$ respectively.

We denote by $\tilde{h}^{S^{*}}$ the pull-back tensor of $h^{S^{*}}$ on $T M \oplus T^{*} M$ :

$$
\tilde{h}^{S^{*}}(\sigma, \tau):=\left(\Phi^{\nabla}\right)^{*}\left(h^{S^{*}}\right)(\sigma, \tau):=h^{S^{*}}\left(\Phi^{\nabla}(\sigma), \Phi^{\nabla}(\tau)\right),
$$

for any $\sigma, \tau \in C^{\infty}\left(T M \oplus T^{*} M\right)$.

Proposition 4.2. If $h$ is a non-degenerate $(0,2)$-tensor field on $M$, then:

$$
\tilde{h}^{S^{*}}(X+\alpha, Y+\beta)=\hat{h}(X+\alpha, Y+\beta)=h(X, Y)+h\left(h^{-1}(\alpha), h^{-1}(\beta)\right),
$$

for any $X, Y \in C^{\infty}(T M)$ and $\alpha, \beta \in C^{\infty}\left(T^{*} M\right)$.

Proof. Let $\sigma=X+\alpha, \tau=Y+\beta, X, Y \in C^{\infty}(T M), \alpha, \beta \in C^{\infty}\left(T^{*} M\right)$. Then:

$$
\begin{gathered}
\tilde{h}^{S^{*}}(\sigma, \tau)=h^{S^{*}}\left(X^{H}+\Phi^{\nabla}(\alpha), Y^{H}+\Phi^{\nabla}(\beta)\right)= \\
=h^{S^{*}}\left(X^{H}, Y^{H}\right)+h^{S^{*}}\left(\Phi^{\nabla}(\alpha), \Phi^{\nabla}(\beta)\right)= \\
=h(X, Y)+h^{S^{*}}\left(\Phi^{\nabla}(\alpha), \Phi^{\nabla}(\beta)\right) .
\end{gathered}
$$

In local coordinates, let $\alpha=\alpha_{k} d x^{k}, \beta=\beta_{l} d x^{l}$ and we get:

$$
\begin{gathered}
h^{S^{*}}\left(\Phi^{\nabla}(\alpha), \Phi^{\nabla}(\beta)\right)=h^{S^{*}}\left(\alpha_{k} \frac{\partial}{\partial y_{k}}, \beta_{l} \frac{\partial}{\partial y_{l}}\right)= \\
=\alpha_{k} \beta_{l} h_{k l}=h\left(h^{-1}(\alpha), h^{-1}(\beta)\right) .
\end{gathered}
$$

Then we get the statement. 


\subsection{Horizontal lift and Sasaki metrics on $T M$}

Let $M$ be a smooth manifold, let $h$ be a non-degenerate $(0,2)$-tensor field on $M$, and let $\nabla$ be an affine connection on $M$. The horizontal lift $h^{H}$ of $h$ on $T M$ with respect to $\nabla$ is defined by:

$$
\begin{gathered}
h^{H}\left(X^{H}, Y^{H}\right)=0 \\
h^{H}\left(X^{V}, Y^{V}\right)=0 \\
h^{H}\left(X^{H}, Y^{V}\right)=h(X, Y),
\end{gathered}
$$

where $X, Y \in C^{\infty}(T M), X^{H}, Y^{H}$ are the horizontal lifts and $X^{V}, Y^{V}$ are the vertical lifts of $X, Y$ respectively.

Let $\pi: T M \rightarrow M$ be the canonical projection and $\pi_{*}: T(T M) \rightarrow T M$ be the tangent map of $\pi$. If $a \in T M$ and $A \in T_{a}(T M)$, then $\pi_{*}(A) \in T_{\pi(a)} M$ and we denote by $\chi_{a}$ the standard identification between $T_{\pi(a)} M$ and its tangent space $T_{a}\left(T_{\pi(a)} M\right)$.

Let $\Psi^{\nabla}: T M \oplus T^{*} M \rightarrow T(T M)$ be the bundle morphism defined by:

$$
\Psi^{\nabla}(X+\alpha):=X_{a}^{H}+\chi_{a}\left(h^{-1}(\alpha)\right)
$$

where $a \in T M$ and $X_{a}^{H}$ is the horizontal lift of $X \in T_{\pi(a)} M$.

Let $\left\{x^{1}, \ldots, x^{n}\right\}$ be local coordinates on $M$, let $\left\{\tilde{x}^{1}, \ldots, \tilde{x}^{n}, y^{1}, \ldots, y^{n}\right\}$ be respectively the corresponding local coordinates on $T M$ and let $\left\{X_{1}, \ldots, X_{n}, \frac{\partial}{\partial y^{1}}, . ., \frac{\partial}{\partial y^{n}}\right\}$ be a local frame on $T(T M)$, where $X_{i}=\frac{\partial}{\partial \tilde{x}^{i}}$. The horizontal lift of $\frac{\partial}{\partial x^{i}}$ is defined by:

$$
\left(\frac{\partial}{\partial x^{i}}\right)^{H}:=X_{i}-y^{k} \Gamma_{i k}^{l} \frac{\partial}{\partial y^{l}}
$$

and we will denote $X_{i}^{H}=:\left(\frac{\partial}{\partial x^{i}}\right)^{H}$. Moreover, the vertical lift of $\frac{\partial}{\partial x^{i}}$ is defined by:

$$
\left(\frac{\partial}{\partial x^{i}}\right)^{V}:=\frac{\partial}{\partial y^{i}},
$$

where $i, k, l$ run from 1 to $n$ and $\Gamma_{i l}^{k}$ are the Christoffel's symbols of $\nabla$.

Let $\Psi^{\nabla}: T M \oplus T^{*} M \rightarrow T(T M)$ be the bundle morphism defined before (which is an isomorphism on the fibres). In local coordinates, we have the following expressions:

$$
\Psi^{\nabla}\left(\frac{\partial}{\partial x^{i}}\right)=X_{i}^{H}
$$




$$
\Psi^{\nabla}\left(d x^{j}\right)=h^{j k} \frac{\partial}{\partial y^{k}}
$$

We denote by $\bar{h}$ the pull-back tensor of $h^{H}$ on $T M \oplus T^{*} M$ :

$$
\bar{h}(\sigma, \tau):=\left(\Psi^{\nabla}\right)^{*}\left(h^{H}\right)(\sigma, \tau):=h^{H}\left(\Psi^{\nabla}(\sigma), \Psi^{\nabla}(\tau)\right)
$$

for any $\sigma, \tau \in C^{\infty}\left(T M \oplus T^{*} M\right)$. Remark that $\bar{h}$ is related to the indefinite metric or to the symplectic structure of $T M \oplus T^{*} M$ as follows.

Proposition 4.3. If $h$ is a symmetric tensor, then:

$$
\bar{h}=-2<\cdot, \cdot>
$$

If $h$ is a skew-symmetric tensor, then:

$$
\bar{h}=-2(\cdot, \cdot) .
$$

Proof. Let $\sigma=X+\alpha, \tau=Y+\beta, X, Y \in C^{\infty}(T M), \alpha, \beta \in C^{\infty}\left(T^{*} M\right)$. Then:

$$
\begin{gathered}
\bar{h}(\sigma, \tau)=h^{H}\left(X^{H}+\Psi^{\nabla}(\alpha), Y^{H}+\Psi^{\nabla}(\beta)\right)= \\
=h^{H}\left(\Psi^{\nabla}(\alpha), Y^{H}\right)+h^{H}\left(X^{H}, \Psi^{\nabla}(\beta)\right) .
\end{gathered}
$$

In local coordinates, let $X=X^{i} \frac{\partial}{\partial x^{i}}, Y=Y^{j} \frac{\partial}{\partial x^{j}}, \alpha=\alpha_{k} d x^{k}, \beta=\beta_{l} d x^{l}$ and we get:

$$
\begin{gathered}
\bar{h}(\sigma, \tau)=h^{H}\left(\alpha_{k} h^{k r} \frac{\partial}{\partial y^{r}}, Y^{j} X_{j}^{H}\right)+h^{H}\left(X^{i} X_{i}^{H}, \beta_{l} h^{l s} \frac{\partial}{\partial y^{s}}\right)= \\
=\alpha_{k} Y^{j} h^{k r} h_{r j}+X^{i} \beta_{l} h^{l s} h_{i s}=\alpha_{k} Y^{j} \delta_{j}^{k} \pm X^{i} \beta_{l} \delta_{i}^{l}= \\
=\alpha(Y) \pm \beta(X),
\end{gathered}
$$

where we denoted by $\delta$ the Kronecker's symbol and the sign + is for $h$ symmetric, - is for $h$ skew-symmetric. Then we get the statement.

The Sasaki $(0,2)$-tensor field $h^{S}$ on $T M$, with respect to $\nabla$, is naturally defined by:

$$
\begin{gathered}
h^{S}\left(X^{H}, Y^{H}\right)=h(X, Y) \\
h^{S}\left(X^{V}, Y^{V}\right)=h(X, Y) \\
h^{S}\left(X^{H}, Y^{V}\right)=0,
\end{gathered}
$$


where $X, Y \in C^{\infty}(T M), X^{H}, Y^{H}$ are the horizontal lifts and $X^{V}, Y^{V}$ are the vertical lifts of $X, Y$ respectively.

We denote by $\bar{h}^{S}$ the pull-back tensor of $h^{S}$ on $T M \oplus T^{*} M$ :

$$
\bar{h}^{S}(\sigma, \tau):=\left(\Psi^{\nabla}\right)^{*}\left(h^{S}\right)(\sigma, \tau):=h^{S}\left(\Psi^{\nabla}(\sigma), \Psi^{\nabla}(\tau)\right)
$$

for any $\sigma, \tau \in C^{\infty}\left(T M \oplus T^{*} M\right)$.

Proposition 4.4. If $h$ is a non-degenerate $(0,2)$-tensor field on $M$, then:

$$
\bar{h}^{S}(X+\alpha, Y+\beta)=\check{h}(X+\alpha, Y+\beta)=h(X, Y)+h\left(h^{-1}(\alpha), h^{-1}(\beta)\right),
$$

for any $X, Y \in C^{\infty}(T M)$ and $\alpha, \beta \in C^{\infty}\left(T^{*} M\right)$.

Proof. Let $\sigma=X+\alpha, \tau=Y+\beta, X, Y \in C^{\infty}(T M), \alpha, \beta \in C^{\infty}\left(T^{*} M\right)$. Then:

$$
\begin{gathered}
\bar{h}^{S}(\sigma, \tau)=h^{S}\left(X^{H}+\Psi^{\nabla}(\alpha), Y^{H}+\Psi^{\nabla}(\beta)\right)= \\
=h^{S}\left(X^{H}, Y^{H}\right)+h^{S}\left(\Psi^{\nabla}(\alpha), \Psi^{\nabla}(\beta)\right)= \\
=h(X, Y)+h^{S}\left(\Psi^{\nabla}(\alpha), \Psi^{\nabla}(\beta)\right) .
\end{gathered}
$$

In local coordinates, let $\alpha=\alpha_{k} d x^{k}, \beta=\beta_{l} d x^{l}$ and we get:

$$
\begin{gathered}
h^{S}\left(\Psi^{\nabla}(\alpha), \Psi^{\nabla}(\beta)\right)=h^{S}\left(\alpha_{k} h^{k r} \frac{\partial}{\partial y^{r}}, \beta_{l} h^{l s} \frac{\partial}{\partial y^{s}}\right)= \\
=\alpha_{k} \beta_{l} h^{k r} h^{l s} h_{r s}=h\left(h^{-1}(\alpha), h^{-1}(\beta)\right) .
\end{gathered}
$$

Then we get the statement.

\subsection{Quasi-statistical structures on cotangent bundles}

Given an affine connection on $M$, the splitting in horizontal and vertical subbundles identifies $T\left(T^{*} M\right)$ with the pull-back bundle $\pi^{*}\left(T M \oplus T^{*} M\right)$, where $\pi: T^{*} M \rightarrow M$ is the canonical projection map. In particular, given a connection on $T M \oplus T^{*} M$, we can define the pull-back connection on $\pi^{*}\left(T M \oplus T^{*} M\right)$.

A direct computation gives the following: 
Proposition 4.5. The pull-back connection $\tilde{\nabla}$ of $\hat{\nabla}$ on $T^{*} M$ is defined, in local coordinates, by:

$$
\begin{gathered}
\tilde{\nabla} \frac{\partial}{\left.\partial x^{i}\right)^{H}}\left(\frac{\partial}{\partial x^{j}}\right)^{H}=\Gamma_{i j}^{k}\left(\frac{\partial}{\partial x^{k}}\right)^{H} \\
\tilde{\nabla} \frac{\partial}{\left(\frac{\partial}{\partial x^{i}}\right)^{H}} \frac{\partial h^{j k}}{\partial y_{j}}=\left(\frac{\partial x^{j}}{\partial l} \Gamma_{i l}^{k}\right) h_{r k} \frac{\partial}{\partial y_{r}} \\
\tilde{\nabla} \frac{\partial}{\partial y_{j}}\left(\frac{\partial}{\partial x^{i}}\right)^{H}=0 \\
\tilde{\nabla} \frac{\partial}{\partial y_{i}} \frac{\partial}{\partial y_{j}}=0 .
\end{gathered}
$$

In local coordinates, the torsion $T^{\tilde{\nabla}}$ of $\tilde{\nabla}$ is:

$$
\begin{gathered}
T^{\tilde{\nabla}}\left(\left(\frac{\partial}{\partial x^{i}}\right)^{H},\left(\frac{\partial}{\partial x^{j}}\right)^{H}\right)=\left(\Gamma_{i j}^{k}-\Gamma_{j i}^{k}\right)\left(\frac{\partial}{\partial x^{k}}\right)^{H}-y_{l} R_{i j k}^{l} \frac{\partial}{\partial y_{k}} \\
T^{\tilde{\nabla}}\left(\frac{\partial}{\partial y_{i}},\left(\frac{\partial}{\partial x^{j}}\right)^{H}\right)=-\left(\left(\frac{\partial h^{i k}}{\partial x^{j}}+h^{i l} \Gamma_{j l}^{k}\right) h_{r k}+\Gamma_{j k}^{i}\right) \frac{\partial}{\partial y_{r}} \\
T^{\tilde{\nabla}}\left(\frac{\partial}{\partial y_{i}}, \frac{\partial}{\partial y_{j}}\right)=0
\end{gathered}
$$

and the curvature $R^{\tilde{\nabla}}$ of $\tilde{\nabla}$, which is the pull-back of $R^{\hat{\nabla}}$, is:

$$
\begin{gathered}
R^{\tilde{\nabla}}\left(\frac{\partial}{\partial y_{i}}, \frac{\partial}{\partial y_{j}}\right)=0 \\
R^{\tilde{\nabla}}\left(\left(\frac{\partial}{\partial x^{i}}\right)^{H}, \frac{\partial}{\partial y_{j}}\right)=0 \\
R^{\tilde{\nabla}}\left(\left(\frac{\partial}{\partial x^{i}}\right)^{H},\left(\frac{\partial}{\partial x^{j}}\right)^{H}\right) \frac{\partial}{\partial y_{k}}=h^{k r} R_{i j r}^{l} h_{l s} \frac{\partial}{\partial y_{s}} \\
R^{\tilde{\nabla}}\left(\left(\frac{\partial}{\partial x^{i}}\right)^{H},\left(\frac{\partial}{\partial x^{j}}\right)^{H}\right)\left(\frac{\partial}{\partial x^{k}}\right)^{H}=\left(R^{\nabla}\left(\frac{\partial}{\partial x^{i}}, \frac{\partial}{\partial x^{j}}\right) \frac{\partial}{\partial x^{k}}\right)^{H} .
\end{gathered}
$$

Therefore we get:

Proposition 4.6. $\nabla$ is flat if and only if $\tilde{\nabla}$ is flat.

THEOREM 4.7. Let $(M, h, \nabla)$ be a quasi-statistical manifold such that $\nabla$ is flat. Then $\left(T^{*} M, h^{S^{*}}, \tilde{\nabla}\right)$ is a flat quasi-statistical manifold. 
Proof. Let us compute $d^{\tilde{\nabla}} h^{S^{*}}$. From the definition of $h^{S^{*}}$ and $\tilde{\nabla}$ we get immediately:

$$
\begin{gathered}
\left(d^{\tilde{\nabla}} h^{S^{*}}\right)\left(\frac{\partial}{\partial y_{i}}, \frac{\partial}{\partial y_{j}}\right)=0 \\
\left(d^{\tilde{\nabla}} h^{S^{*}}\right)\left(\left(\frac{\partial}{\partial x^{i}}\right)^{H}, \frac{\partial}{\partial y_{j}}\right)=0 \\
\left(d^{\tilde{\nabla}} h^{S^{*}}\right)\left(\left(\frac{\partial}{\partial x^{i}}\right)^{H},\left(\frac{\partial}{\partial x^{j}}\right)^{H}\right)\left(\frac{\partial}{\partial y_{k}}\right)=-y_{l} R_{i j r}^{l} h^{k r} \\
\left(d^{\tilde{\nabla}} h^{S^{*}}\right)\left(\left(\frac{\partial}{\partial x^{i}}\right)^{H},\left(\frac{\partial}{\partial x^{j}}\right)^{H}\right)\left(\frac{\partial}{\partial x^{k}}\right)^{H}=\left(d^{\nabla} h\right)\left(\frac{\partial}{\partial x^{i}}, \frac{\partial}{\partial x^{j}}\right)\left(\frac{\partial}{\partial x^{k}}\right) .
\end{gathered}
$$

Then we get the statement.

Moreover, considering the Patterson-Walker metric, $\tilde{h}_{ \pm}$, we get the following:

THEOREM 4.8. Let $(M, h, \nabla)$ be a quasi-statistical manifold such that $\nabla$ is flat. Then $\left(T^{*} M, \tilde{h}_{ \pm}, \tilde{\nabla}\right)$ is a quasi-statistical manifold.

ProOF. Let us compute $d^{\tilde{\nabla}} \tilde{h}_{ \pm}$. From the definition of $\tilde{h}_{ \pm}$and $\tilde{\nabla}$ we get immediately:

$$
\begin{gathered}
\left(d^{\tilde{\nabla}} \tilde{h}_{ \pm}\right)\left(\frac{\partial}{\partial y_{i}}, \frac{\partial}{\partial y_{j}}\right)=0 \\
\left(d^{\tilde{\nabla}} \tilde{h}_{ \pm}\right)\left(\left(\frac{\partial}{\partial x^{i}}\right)^{H}, \frac{\partial}{\partial y_{j}}\right)=0 \\
\left(d^{\tilde{\nabla}} \tilde{h}_{ \pm}\right)\left(\left(\frac{\partial}{\partial x^{i}}\right)^{H},\left(\frac{\partial}{\partial x^{j}}\right)^{H}\right)\left(\frac{\partial}{\partial x^{k}}\right)^{H}=-y_{l} R_{i j k}^{l} \\
\left(d^{\nabla} \tilde{h}_{ \pm}\right)\left(\left(\frac{\partial}{\partial x^{i}}\right)^{H},\left(\frac{\partial}{\partial x^{j}}\right)^{H}\right)\left(\frac{\partial}{\partial y_{k}}\right)= \pm h^{k l}\left(d^{\nabla} h\right)\left(\frac{\partial}{\partial x^{i}}, \frac{\partial}{\partial x^{j}}\right)\left(\frac{\partial}{\partial x^{l}}\right)
\end{gathered}
$$

where the sign + is for $h$ symmetric, - is for $h$ skew-symmetric. Then we get the statement.

Definition 4.9. A quasi-statistical manifold $(M, h, \nabla)$ such that $\nabla$ is flat is called a Hessian manifold.

Therefore we get:

Corollary 4.10. If $(M, h, \nabla)$ is a Hessian manifold, then $\left(T^{*} M, h^{S^{*}}, \tilde{\nabla}\right)$ and $\left(T^{*} M, \tilde{h}_{ \pm}, \tilde{\nabla}\right)$ are Hessian manifolds. 


\subsection{Quasi-statistical structures on tangent bundles}

Given a non-degenerate $(0,2)$-tensor field $h$ on $M$, we have an isomorphism between $T\left(T^{*} M\right)$ and $T(T M)$. The connection $\tilde{\tilde{\nabla}}$ on $T M$ corresponding to $\tilde{\nabla}$ on $T^{*} M$, is the following:

$$
\begin{gathered}
\tilde{\nabla}_{\left(\frac{\partial}{\partial x^{i}}\right)^{H}}\left(\frac{\partial}{\partial x^{j}}\right)^{H}=\Gamma_{i j}^{k}\left(\frac{\partial}{\partial x^{k}}\right)^{H} \\
\tilde{\tilde{\nabla}}{ }_{\left(\frac{\partial}{\partial x^{i}}\right)^{H}} \frac{\partial}{\partial y^{j}}=\Gamma_{i j}^{k} \frac{\partial}{\partial y^{k}} \\
\tilde{\tilde{\nabla}} \frac{\partial}{\partial y^{j}}\left(\frac{\partial}{\partial x^{i}}\right)^{H}=0 \\
\tilde{\tilde{\nabla}}{\frac{\partial}{\partial y^{i}}}^{\frac{\partial}{\partial y^{j}}}=0 .
\end{gathered}
$$

In local coordinates, the torsion $T^{\tilde{\tilde{\nabla}}}$ of $\tilde{\tilde{\nabla}}$ is:

$$
\begin{gathered}
T^{\tilde{\tilde{\nabla}}}\left(\left(\frac{\partial}{\partial x^{i}}\right)^{H},\left(\frac{\partial}{\partial x^{j}}\right)^{H}\right)=\left(\Gamma_{i j}^{k}-\Gamma_{j i}^{k}\right)\left(\frac{\partial}{\partial x^{k}}\right)^{H}-y^{l} R_{i j l}^{k} \frac{\partial}{\partial y^{k}} \\
T^{\tilde{\tilde{\nabla}}}\left(\frac{\partial}{\partial y^{i}},\left(\frac{\partial}{\partial x^{j}}\right)^{H}\right)=0 \\
T^{\tilde{\tilde{\nabla}}}\left(\frac{\partial}{\partial y^{i}}, \frac{\partial}{\partial y^{j}}\right)=0
\end{gathered}
$$

and the curvature $R^{\tilde{\tilde{\nabla}}}$ of $\tilde{\tilde{\nabla}}$ is:

$$
\begin{gathered}
R^{\tilde{\tilde{\nabla}}}\left(\frac{\partial}{\partial y^{i}}, \frac{\partial}{\partial y^{j}}\right)=0 \\
R^{\tilde{\nabla}}\left(\left(\frac{\partial}{\partial x^{i}}\right)^{H}, \frac{\partial}{\partial y^{j}}\right)=0 \\
R^{\tilde{\nabla}}\left(\left(\frac{\partial}{\partial x^{i}}\right)^{H},\left(\frac{\partial}{\partial x^{j}}\right)^{H}\right) \frac{\partial}{\partial y^{k}}=R_{i j k}^{l} \frac{\partial}{\partial y^{l}} \\
R^{\tilde{\nabla}}\left(\left(\frac{\partial}{\partial x^{i}}\right)^{H},\left(\frac{\partial}{\partial x^{j}}\right)^{H}\right)\left(\frac{\partial}{\partial x^{k}}\right)^{H}=R^{\nabla}\left(\frac{\partial}{\partial x^{i}}, \frac{\partial}{\partial x^{j}}\right) \frac{\partial}{\partial x^{k}}
\end{gathered}
$$

Therefore we get:

Proposition 4.11. $\nabla$ is flat if and only if $\tilde{\tilde{\nabla}}$ is flat. 
THEOREM 4.12. Let $(M, h, \nabla)$ be a quasi-statistical manifold such that $\nabla$ is flat. Then $\left(T M, h^{S}, \tilde{\tilde{\nabla}}\right)$ is a flat quasi-statistical manifold.

Proof. Let us compute $d^{\tilde{\nabla}} h^{S}$. From the definition of $h^{S}$ and $\tilde{\tilde{\nabla}}$ we get immediately:

$$
\begin{gathered}
\left(d^{\tilde{\nabla}} h^{S}\right)\left(\frac{\partial}{\partial y^{i}}, \frac{\partial}{\partial y^{j}}\right)=0 \\
\left(d^{\tilde{\nabla}} h^{S}\right)\left(\left(\frac{\partial}{\partial x^{i}}\right)^{H}, \frac{\partial}{\partial y^{j}}\right)=0 \\
\left(d^{\tilde{\nabla}} h^{S}\right)\left(\left(\frac{\partial}{\partial x^{i}}\right)^{H},\left(\frac{\partial}{\partial x^{j}}\right)^{H}\right)\left(\frac{\partial}{\partial y^{k}}\right)=-y^{l} R_{i j l}^{r} h_{r k} \\
\left(d^{\tilde{\nabla}} h^{S}\right)\left(\left(\frac{\partial}{\partial x^{i}}\right)^{H},\left(\frac{\partial}{\partial x^{j}}\right)^{H}\right)\left(\frac{\partial}{\partial x^{k}}\right)^{H}=\left(d^{\nabla} h\right)\left(\frac{\partial}{\partial x^{i}}, \frac{\partial}{\partial x^{j}}\right)\left(\frac{\partial}{\partial x^{k}}\right) .
\end{gathered}
$$

Then we get the statement.

Moreover, considering the horizontal lift metric, $h^{H}$, we get the following:

THEOREM 4.13. Let $(M, h, \nabla)$ be a quasi-statistical manifold such that $\nabla$ is flat. Then $\left(T M, h^{H}, \tilde{\nabla}\right)$ is a quasi-statistical manifold if and only if $\nabla h=0$.

Proof. Let us compute $d^{\tilde{\nabla}} h^{H}$. From the definition of $h^{H}$ and $\tilde{\tilde{\nabla}}$ we get immediately:

$$
\begin{gathered}
\left(d^{\tilde{\nabla}} h^{H}\right)\left(\frac{\partial}{\partial y^{i}}, \frac{\partial}{\partial y^{j}}\right)=0 \\
\left(d^{\tilde{\tilde{\nabla}}} h^{H}\right)\left(\left(\frac{\partial}{\partial x^{i}}\right)^{H}, \frac{\partial}{\partial y^{j}}\right)= \pm\left(\nabla_{\left.\frac{\partial}{\partial x^{i}} h\right) \frac{\partial}{\partial x^{j}}}\right. \\
\left(d^{\tilde{\nabla}} h^{H}\right)\left(\left(\frac{\partial}{\partial x^{i}}\right)^{H},\left(\frac{\partial}{\partial x^{j}}\right)^{H}\right)\left(\frac{\partial}{\partial x^{k}}\right)^{H}=-y^{l} R_{i j l}^{s} h_{s k} \\
\left(d^{\tilde{\nabla}} h^{H}\right)\left(\left(\frac{\partial}{\partial x^{i}}\right)^{H},\left(\frac{\partial}{\partial x^{j}}\right)^{H}\right)\left(\frac{\partial}{\partial y^{k}}\right)=\left(d^{\nabla} h\right)\left(\frac{\partial}{\partial x^{i}}, \frac{\partial}{\partial x^{j}}\right)\left(\frac{\partial}{\partial x^{k}}\right),
\end{gathered}
$$

where the sign + is for $h$ symmetric, - is for $h$ skew-symmetric. Then we get the statement.

Therefore we get:

Corollary 4.14. If $(M, h, \nabla)$ is a Hessian manifold, then $\left(T M, h^{S}, \tilde{\nabla}\right)$ is a Hessian manifold. Moreover, if $\nabla h=0$, then $\left(T M, h^{H}, \tilde{\tilde{\nabla}}\right)$ is a Hessian manifold. 


\section{Norden and Para-Norden structures on cotangent and tangent bundles}

Norden manifolds, also called almost complex manifolds with B-metric, were introduced in [8]. They have applications in mathematics and in theoretical physics.

Definition 5.1. A Norden manifold, $(M, J, h)$, is an almost complex manifold $(M, J)$ with a pseudo-Riemannian metric, $h$ (called Norden metric), such that $J$ is $h$-symmetric.

Moreover, if $J$ is integrable, then $(M, J, h)$ is called complex Norden manifold.

Definition 5.2. An almost Para-complex Norden manifold (or simply, almost ParaNorden manifold $),(M, J, h)$, is a real even dimensional smooth manifold $M$ with a pseudoRiemannian metric, $h$, and a $(1,1)$-tensor field, $J$, such that $J^{2}=I$, the two eigenbundles $T^{+} M, T^{-} M$, associated to the two eigenvalues $+1,-1$, of $J$ respectively have the same rank and $J$ is $h$-symmetric.

Moreover, if $J$ is integrable, then $(M, J, h)$ is called Para-Norden manifold.

\subsection{Norden and Para-Norden structures on cotangent bundles}

Let $(M, h)$ be a pseudo-Riemannian manifold and let $\hat{J}_{c}, \hat{J}_{p}$ be the generalized complex structure and the generalized product structure defined by $h$ in (1) and (2) respectively. Again we will denote $\hat{J}_{\mp}$ for $\hat{J}_{c}=: \hat{J}_{-}$and $\hat{J}_{p}=: \hat{J}_{+}$.

Let $\nabla$ be an affine connection on $M$ and let $\Phi^{\nabla}: T M \oplus T^{*} M \rightarrow T\left(T^{*} M\right)$ be the bundle morphism defined by (8). We define:

$$
\tilde{J}_{\mp}^{\nabla}=: \Phi^{\nabla} \circ \hat{J}_{\mp} \circ\left(\Phi^{\nabla}\right)^{-1}
$$

We have immediately that $\left(\tilde{J}_{\mp}^{\nabla}\right)^{2}=\mp I$.

Proposition 5.3. Let $\tilde{h}$ be the Patterson-Walker metric on $T^{*} M$. Then $\left(T^{*} M, \tilde{J}_{-}^{\nabla}, \tilde{h}\right)$ is a Norden manifold and $\left(T^{*} M, \tilde{J}_{+}^{\nabla}, \tilde{h}\right)$ is an almost Para-Norden manifold. Moreover, if $(M, h, \nabla)$ is a flat quasi-statistical manifold, then $\left(T^{*} M, \tilde{J}_{-}^{\nabla}, \tilde{h}\right)$ is a complex Norden manifold and $\left(T^{*} M, \tilde{J}_{+}^{\nabla}, \tilde{h}\right)$ is a Para-Norden manifold.

Proof. In local coordinates, we get the following:

$$
\tilde{J}_{\mp}^{\nabla}\left(X_{i}^{H}\right)=: h_{i k} \frac{\partial}{\partial y_{k}}
$$




$$
\tilde{J}_{\mp}^{\nabla}\left(\frac{\partial}{\partial y_{j}}\right)=: \mp h^{j k} X_{k}^{H} .
$$

In particular, we have:

$$
\begin{gathered}
\tilde{h}\left(\tilde{J}_{\mp}^{\nabla}\left(X_{i}^{H}\right), X_{j}^{H}\right)=h_{i j} \\
\tilde{h}\left(\tilde{J}_{\mp}^{\nabla}\left(\frac{\partial}{\partial y_{i}}\right), \frac{\partial}{\partial y_{j}}\right)=\mp h^{i j} \\
\tilde{h}\left(\tilde{J}_{\mp}^{\nabla}\left(X_{i}^{H}\right), \frac{\partial}{\partial y_{j}}\right)=0 \\
\tilde{h}\left(\tilde{J}_{\mp}^{\nabla}\left(\frac{\partial}{\partial y_{i}}\right), X_{j}^{H}\right)=0,
\end{gathered}
$$

therefore, from the symmetry of $h$, we get the first statement.

Moreover, if we compute the Nijenhuis tensor field of $\tilde{J}_{\mp}^{\nabla}$, we have:

$$
\begin{gathered}
N_{\tilde{J}_{\mp}^{\nabla}}\left(X_{i}^{H}, X_{j}^{H}\right)= \pm\left(h^{k l}\left(d^{\nabla} h\right)\left(\frac{\partial}{\partial x^{i}}, \frac{\partial}{\partial x^{j}}\right)\left(\frac{\partial}{\partial x^{k}}\right)-y_{k} R_{i j l}^{k}\right) \frac{\partial}{\partial y_{l}} \\
N_{\tilde{J}_{\mp}^{\nabla}}\left(X_{i}^{H}, \frac{\partial}{\partial y_{j}}\right)=h^{j l}\left(h^{s r} y_{k} R_{i l s}^{k} X_{r}^{H} \mp\left(d^{\nabla} h\right)\left(\frac{\partial}{\partial x^{i}}, \frac{\partial}{\partial x^{l}}\right)\left(\frac{\partial}{\partial x^{r}}\right) \frac{\partial}{\partial y_{r}}\right) \\
N_{\tilde{J}_{\mp}^{\nabla}}\left(\frac{\partial}{\partial y_{i}}, \frac{\partial}{\partial y_{j}}\right)=h^{i k} h^{j l}\left(h^{p s}\left(d^{\nabla} h\right)\left(\frac{\partial}{\partial x^{l}}, \frac{\partial}{\partial x^{k}}\right)\left(\frac{\partial}{\partial x^{p}}\right) X_{s}^{H}+y_{s} R_{k l r}^{s} \frac{\partial}{\partial y^{r}}\right) .
\end{gathered}
$$

Then the proof is complete.

REMARK 5.4. If $h$ is a non-degenerate skew-symmetric $(0,2)$-tensor field on $M$, then the same construction gives rise to a Hermitian, respectively Para-Hermitian, structure on $T^{*} M$.

\subsection{Norden and Para-Norden structures on tangent bundles}

Let $(M, h)$ be a pseudo-Riemannian manifold and let $\hat{J}_{c}, \hat{J}_{p}$ be the generalized complex structure and the generalized product structure defined by $h$ in (1) and (2) respectively. Again we will denote $\hat{J}_{\mp}$ for $\hat{J}_{c}=: \hat{J}_{-}$and $\hat{J}_{p}=: \hat{J}_{+}$.

Let $\nabla$ be an affine connection on $M$ and let $\Psi^{\nabla}: T M \oplus T^{*} M \rightarrow T(T M)$ be the bundle morphism defined by (9). We define:

$$
\bar{J}_{\mp}^{\nabla}=: \Psi^{\nabla} \circ \hat{J}_{\mp} \circ\left(\Psi^{\nabla}\right)^{-1}
$$


Let $X \in C^{\infty}(T M)$ and let $X^{H}, X^{V}$ be respectively the horizontal and vertical lift of $X$. We have immediately that

$$
\begin{gathered}
\bar{J}_{\mp}^{\nabla}\left(X^{H}\right)=X^{V} \\
\bar{J}_{\mp}^{\nabla}\left(X^{V}\right)=\mp X^{H} .
\end{gathered}
$$

A direct computation gives the following:

Proposition 5.5. Let $h^{H}$ be the horizontal lift metric of $h$ on TM. Then $\left(T M, \bar{J}_{-}^{\nabla}, h^{H}\right)$ is a Norden manifold and $\left(T M, \bar{J}_{+}^{\nabla}, h^{H}\right)$ is an almost Para-Norden manifold.

REMARK 5.6. The almost complex structure $\bar{J}_{-}^{\nabla}$ is the canonical almost complex structure of $T M$ defined in [3. In particular, it is integrable if and only if $\nabla$ is flat and torsion-free.

\section{References}

[1] S. Amari, Differential-Geometrical Methods in Statistics, Lecture Notes in Statistics, 28 Springer (1985).

[2] A. M. Blaga, A. Nannicini, Generalized metallic structures, arXiv:1807.08308, 2018.

[3] P. Dombrowski, On the geometry of tangent bundles, J. reine angew. Math. 210 (1962), 73-88.

[4] H. Matsuzoe, Quasi-statistical manifolds and geometry of affine distributions, Pure and Applied Differential Geometry 2012: In Memory of Franki Dillen, Berichte aus der Mathematik, ed. Joeri Van der Veken, Ignace Van de Woestyne, Leopold Verstraelen, Luc Vrancken, Shaker Verlag, 2013.

[5] A. Nannicini, Almost complex structures on cotangent bundles and generalized geometry, J. Geom. Phys. 60 (2010), 1781-1791.

[6] A. Nannicini, Calibrated complex structures on the generalized tangent bundle of a Riemannian manifold, J. Geom. Phys. 56 (2006), 903-916.

[7] M. Nogushi, Geometry of statistical manifolds, Differ. Geom. Appl. 2 (1992), 197-222.

[8] A. P. Norden, On a class of four-dimensional A-spaces, Russian Math. (Izv VUZ) 17 (4) (1960), 145-157. 
[9] E. M. Patterson, A. G. Walker, Riemann extension, Quart. J. Math. Oxford 3 (1952), 19-28.

Adara M. Blaga

Department of Mathematics

West University of Timişoara

Bld. V. Pârvan nr. 4, 300223, Timişoara, România

adarablaga@yahoo.com

Antonella Nannicini

Department of Mathematics and Informatics "U. Dini"

University of Florence

Viale Morgagni, 67/a, 50134, Firenze, Italy

antonella.nannicini@unifi.it 